\title{
Ecotourism development and the heterogeneity of tourists
}

\author{
Joung Hun Lee ${ }^{1} \cdot$ Yoh Iwasa ${ }^{2}$
}

Received: 12 December 2019 / Accepted: 30 March 2020 / Published online: 29 April 2020

(C) The Author(s) 2020

\begin{abstract}
Ecotourism is potentially capable of making biodiversity conservation and ecosystem management economically feasible. Here, we propose a simple model for ecotourism development considering the heterogeneity of tourists, motivated by the case of Jeju Island, South Korea. We analyze the optimal investment in accommodation capacity (i.e., hotels, restaurants, and transportation) and in improving the quality of the environment (i.e., biodiversity, landscape, and cultural activities). "General tourists" are abundant but will not return to the focal site, and their future number is unpredictable, while "loyal tourists" may return to the site if they are attracted by its environmental and/or cultural assets. The analysis shows that the economically profitable policy is either the one with a large investment in accommodation capacity targeting general tourists or the one with a large investment to environmental quality targeting loyal tourists, but an intermediate mixture of these extremes cannot be the optimal. We also discuss "zoning," in which a site is separated to two areas, and many visitors stay in the first area and visit the second area to enjoy the high-quality environment.
\end{abstract}

Keywords Ecotourism $\cdot$ General tourists $\cdot$ Loyal tourists $\cdot$ Optimistic policy $\cdot$ Pessimistic policy

\section{Introduction}

For the success of biodiversity conservation and ecosystem management, securing a stable supply of funds to cover maintenance costs is always an important issue. Ecotourism is a hopeful method to achieve this goal, although there can be many problems. Ecotourists come to the site to enjoy the biodiversity, landscape, history, and cultural activities of the local area. Investments in the restoration of local ecosystems and the protection of endangered species and endemic biota, as well as in improvements to the natural landscape and the construction of facilities for visitor activities (e.g., walking paths, museums, bookstores) may enhance the value of the site to ecotourists. Sites may also promote cultural activities and hire rangers and curators to study the site's unique

Yoh Iwasa

yohiwasa@kyudai.jp

1 Department of Biology, Faculty of Science, Kyushu University, Motooka 744, Nishi-ku, Fukuoka 819-0395, Japan

2 Department of Bioscience, School of Science and Technology, Kwansei Gakuin University, Gakuen 2-1, Sanda, Hyogo 669-1337, Japan natural and cultural features, administer the site, and give lectures to visitors on topics on which they have special knowledge. Because ecotourism is an important means by which a local community can achieve sustainable development and conserve its natural ecosystem and biodiversity, theoretical study of the basis of successful ecotourism is an important research theme.

In fact, tourism in general has become one of the most rapidly increasing industries in the contemporary world with growing importance to economies worldwide (UN World Tourism Organization [UNWTO] 2011, 2018; World Bank 2016; World Travel and Tourism Council [WTTC] 2016). Ecotourism is one type of tourism that emphasizes local biodiversity, ecosystems, natural history, and landscape, as well as the cultural assets of sites and regions (Ceballos-Lascuráin 1996; Lee and Iwasa 2011). With the growing popularity of ecotourism, attention has increasingly focused on the need to define the responsibilities of local residents in terms of environmentally friendly management and sustainable development of popular destinations (Goodwin 1996; Torquebiau and Taylor 2009).

As the competition among tourism sites has become more intense, some sites have faced large fluctuations in the number of visitors. Numerous tourists come for a while, but then, their numbers may suddenly drop for various reasons, such as the 
emergence of a more attractive competing tourist spot, a new tourism trend, or political issues (Kim 2018; Park et al. 2019). Tourism sites that have invested to increase accommodation and transportation capacities can suffer from such unexpected fluctuations in the number of tourists (Latzko 2004; Luthe and Wyss 2014). We may call these as "general tourists."

Alternatively, tourism development can target "loyal tourists," people who become fond of the site and are likely to return to it many times in the future. A typical example of this type of development is ecotourism.

The heterogeneity of tourists can be illustrated by the case of Jeju Island, Republic of Korea, which is the largest island in the Korean Strait off the Korean Peninsula. It is a popular tourist destination and includes a UNESCO World Natural Heritage Site, Global Geopark, and Biosphere Reserve, which in June 2019 was extended to encompass the whole area of the island. Owing to the island's unique natural environment, tourism has become one of its main industries, accounting for about $10 \%$ of the gross regional domestic product of the island (Jeju Branch of the Bank of Korea 2018). This island of some 600,000 residents (as of 2015) has received a remarkable annual tourist volume of over 10 million since 2013, owing to its established reputation as a nature-based destination and the establishment of service by low-cost carriers to the island (Chung and Whang 2011; Kang et al. 2012).

A survey conducted on Jeju Island reports two distinctive types of tourists: cruise tourists seem to be general tourists, and Do-It Yourself (DIY) tourists seem to be loyal tourists in our terminology. Most cruise travelers are first-time visitors, and they rely mainly on travel agents and travel brochures $(60 \%)$ to plan their trip. In contrast, first-time visitors account for only $30 \%$ of DIY travelers, and their main information source $(50 \%)$ is the Internet, but they also use direct or indirect information sources, such as the recommendations of close friends and their own experiences from previous visits (Jeju Special Self-Governing Province 2017). The Internet has been widely adopted for travel planning (Kim et al. 2014; Xiang et al. 2014, 2015), and social media and sites for sharing photos and videos have become very popular for online trip planning (Xiang et al. 2014, 2015).

In recent years, Jeju Island has sought to expand the number of visitors, especially from countries in East Asia, but the rapid increase in the number of tourists has caused environmental problems that threaten sustainable development on the island (Suh and Jo 2015; Choi and Suh 2019). Jeju Island also experienced a sudden drop in the number of tourists, mainly caused by international tensions (An et al. 2017; Kim and Kim 2017; Park et al. 2019), which illustrates the problem of relying on general tourists. On the other hand, Jeju Island has many natural attractions, such as the cone-shaped parasitic volcanoes, called oreums that are distributed across the island and managed by ecotourism programs to enhance both their conservation and the local economy ( $\mathrm{Ki}$ et al. 2016). In addition, Jeju has many cultural sites associated with its long eventful history that might attract tourists. Therefore, Jeju is an excellent candidate for tourism focusing on loyal tourists.

In this paper, we discuss ecotourism development policy by considering two different types of tourists: general tourists and loyal tourists. General tourists may come in large numbers, but in the future, they might be attracted to other tourism sites. Here, we consider the future number of general tourists to be unpredictable - the number might remain high in the future, but it can become low with some probability. In contrast, loyal tourists are interested in the regional landscape, biodiversity, and local culture. Their number may increase if these qualities of the tourism site are improved, but it decreases if the number of hotels and other accommodation facilities increases. The number of tourists that a site can accept is limited by the capacity of accommodations, such as hotels and restaurants. We discuss the optimal policy to maximize the mean future net benefit to the local community. One possible strategy to achieve this aim is to increase the accommodation capacity (e.g., the number of hotel rooms), and another is to improve the quality of the tourism site; some mix of the two strategies is also possible. The analysis indicates that there are often two distinct peaks of net benefit, one resulting from the first strategy (increasing the capacity of accommodations), and the other from the second (improving site quality), and that mixing these two strategies is unlikely to be beneficial. We also discuss the possibility of site zoning. The tourism site is separated into one zone with many hotels and other facilities, and the other with a limited number of hotels and other accommodations but an improved environment. Many visitors may stay in the first zone and visit the second zone to enjoy the highquality environment, if the two are not very far apart.

\section{Investments when there are only loyal tourists}

Before analyzing the full model, we first consider a simpler situation in which there are only loyal tourists. Let $x$ be the number of potential loyal tourists, who are willing to come to the focal site if the capacity allows. The number of realized tourists is constrained by the accommodation capacity, which includes the number of hotel rooms, restaurants, and other facilities. Let $C$ be the capacity of the site and $R_{x}$ be the realized number of tourists who are accepted as visitors to the site.

$R_{x}=\min [C, x]$

where the min function indicates the smaller of the two quantities.

The potential number of loyal tourist changes over time and depends on the experiences of loyal tourists, who are assumed to disseminate their level of satisfaction via social 
networks and other methods as well as to actively use such methods for their own trip planning.

Let $W(C, Q)$ be the level of satisfaction of loyal tourists. It decreases with accommodation capacity $C$ and increases with investment in the quality of the site, which is denoted by $Q$. Specifically, we assume the following inequalities:

$\frac{\partial W}{\partial C}<0, \frac{\partial^{2} W}{\partial C^{2}}>0, \frac{\partial W}{\partial Q}>0$, and $\frac{\partial^{2} W}{\partial Q^{2}}<0$.

As the function illustrating the examples in this paper, we adopt $W(C, Q)=w_{o} e^{-m C} \sqrt{Q}$. Since we would like to focus on between-year change, rather than seasonal fluctuations within a year, we adopted the discrete-time logistic equation for the potential number of loyal tourists:

$\Delta x=\delta x\{W(C, Q)-x\}$.

We here model the dynamics of the number of loyal tourists based on the experience before which takes more than a few years to exhibit the changes. Hence, the number of potential loyal tourists $x$ eventually converges on $W(C$, $Q$ ). In addition, we assume that $\delta$ is positive but not very large, and hence, the dynamics show smooth convergence to the final value $W(C, Q)$. If $\delta$ was large, the dynamics could generate overshooting the target level, or periodic, or even chaotic fluctuations. Such a quick response to the boom and fashion in the site choice would be typical to general tourists, but not for loyal tourists. We assume that the response of the potential number of loyal tourists take more than a few years to build up their preference.

The economic benefit to the local community that chooses to develop the site for tourism is $b \cdot R_{x}=b \cdot \min [C, W(C, Q)]$, where $W(C, Q)$ is a decreasing function of $C$. Figure 1a illustrates two functions: $C$ and $W(C, Q)$. The maximum economic benefit under the constraint of accommodation capacity is achieved when $C=W(C, Q)$ holds.

To improve the focal tourism site, two policy options are available: one option is to increase the capacity $C$ by allowing hotels and other facilities to be constructed. This option is accompanied by cost $k_{C}$ per unit increase in $C$, where $k_{C}$ includes not only the costs of constructing and maintaining buildings, roads, and other transportation systems but also those of the resulting environmental burden and its mitigation. The second option is to increase the quality of the tourism site: unit increases in the quality parameter $Q$ are accompanied by $\operatorname{cost} k_{Q}$. Improvements in quality may include the restoration of forests, grasslands, and other original ecosystems, measures taken to preserve endangered species, the construction of museums, other educational facilities, or a theater for local cultural events, performing research, publishing books, and generally encouraging people to take an interest in the environment, culture, and history of the local area.
Then, the expected net benefit $\phi$ is calculated as follows:

$\phi=b \cdot \min [\mathrm{C}, W(C, Q)]-k_{C} C-k_{Q} Q$.

Here, we assume that $b>k_{C}$, which implies that the economic benefit derived from capacity enhancement is higher than the cost when the potential number of loyal tourists is greater than the capacity. Under this assumption, $\phi$ reaches a maximum when $C=W(C, Q)$. On the $(Q, C)$ plane, the graph of this function appears as a curve with a positive slope (Fig. 1b). The optimal strategy for the community must lie on this curve.

To determine the optimal choice for $Q$, we treat $C$ as an increasing function of $Q$. Then, by setting the derivative of Eq. (4) with respect to $Q$ equal to zero, we obtain

$\frac{d C}{d Q}=k_{Q} /\left(b-k_{C}\right)$.

Eq. (5a) describes the slope of the curve drawn in the $(Q, C)$ plane at the optimal point (Fig. 1b). Because $C=W(C$, $Q$ ), we can rewrite Eq. (5a) as,

$\left(b-k_{C}\right) \frac{\partial W}{\partial Q}=k_{Q}\left(1-\frac{\partial W}{\partial C}\right)$,

Eq. (5b) is useful for calculating the solution mathematically. Figure $1 \mathrm{~b}$ indicates that the optimal choice of $C$ and $Q$ is controlled by the cost coefficient for quality improvement $k_{Q}$. When $k_{Q}$ is small, both $C$ and $Q$ are large, implying that large investments in both environmental quality improvement and capacity are beneficial (Fig. 1b). In contrast, if $k_{Q}$ is large, it is not efficient to invest in environmental quality to attract loyal tourists; hence, the optimal investments in $C$ and $Q$ are both small. This result implies that we should focus quality enhancement efforts on only those tourism sites where a small investment in environmental quality can result in a rapid increase in their attractiveness.

\section{Investment when there are general tourists and loyal tourists}

The situation is quite different when there are general tourists in addition to loyal tourists. Because general tourists come in large numbers, they are an attractive target of tourism investment, although they may stop coming in the future with some probability. In addition, the same tourists are unlikely to return to the site; thus, the future numbers of general tourists, unlike those of loyal tourists, are independent of the experience of the current tourists. General tourists compete with loyal tourists for hotel vacancies and other accommodations if facilities are limited. 
Fig. 1 Analysis for a tourist population that includes loyal tourists only. a Horizontal axis is accommodation capacity $C$, and vertical axis is a realized number of visitors to a tourism site $R_{x}$. $W(C, Q)$, which represents the potential number of loyal tourists at equilibrium, and function $R_{X}=$ $C$ (a straight line) is plotted. The realized number of visitors is the smaller parts of the two functions, and the maximum number of visitors is realized where the two curves described by these functions intersect. Other parameters are $w_{0}=10, m=$ 0.03 , and $Q=15 . \mathbf{b}$ Optimal choices of $C$ (investment in accommodation capacity) and $Q$ (investment in environmental quality). The curve is $C=W(Q$, $C)$. Eq. (5a) indicates that as $k_{Q}$ decreases, both $C$ and $Q$ decrease. Other parameters are $w_{0}=2, m=$ $0.1, b=5$, and $k_{C}=0.1$ a

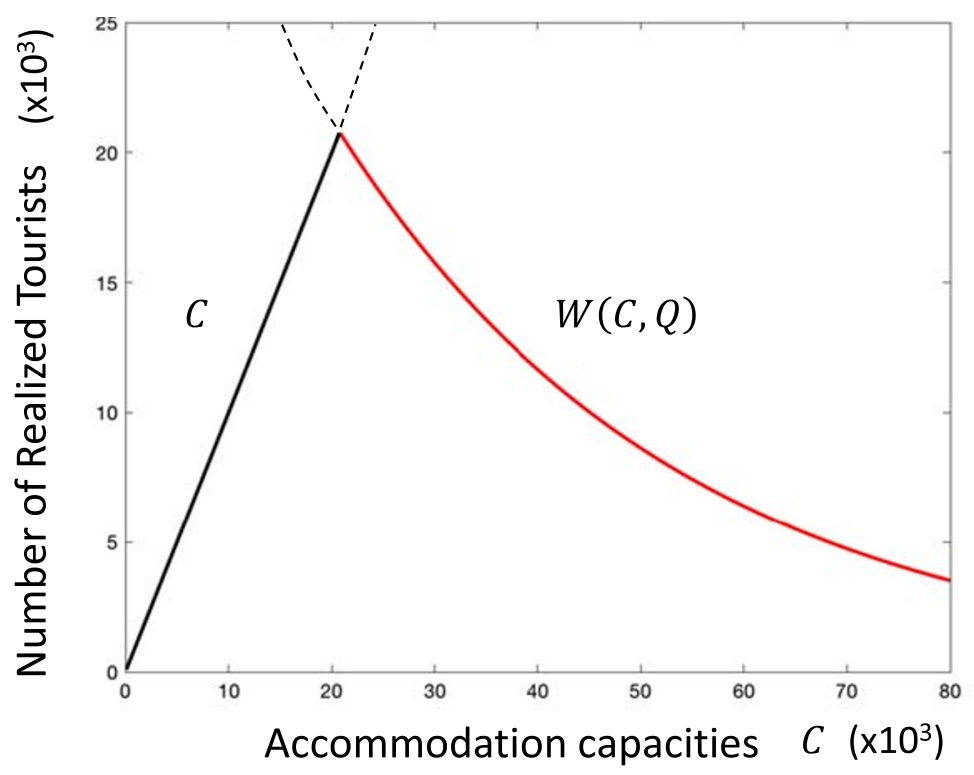

b

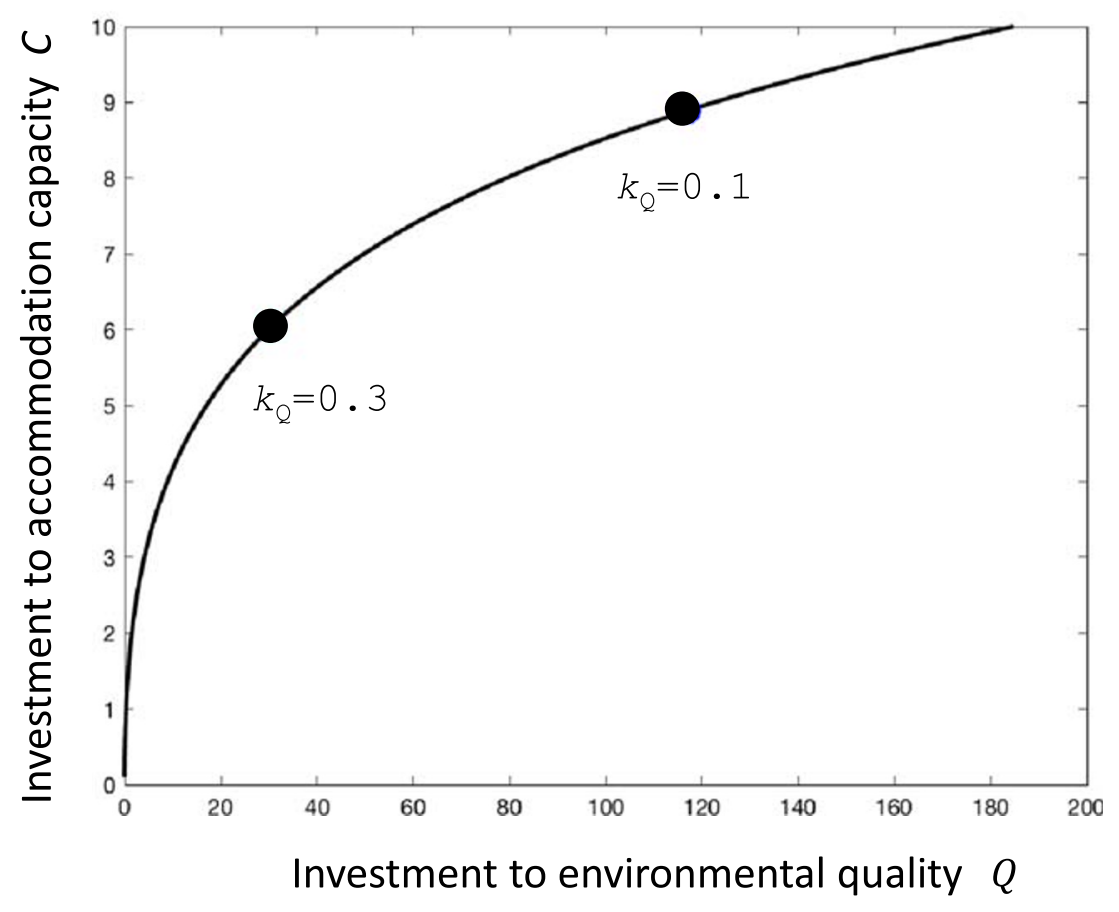

Let $y$ be the number of general tourists in the future. This number may include large stochasticity. Let $N_{h}$ and $N_{l}$ be the high and low numbers of general tourists, where $N_{h} \gg N_{l}>0$. We assume that

$y=N_{h} \quad$ with probability $1-p$, and

$y=N_{l} \quad$ with probability $p$,

where $p$ is the probability of a general tourist "crash."

Because there is a capacity limitation, the economic benefit is $b \cdot \min [C, x+y]$. If the total number of potential tourists, 
including both loyal tourists and general tourists, is less than the capacity of the tourism site, all of the people who are willing to come to the site will be accepted. However, if the sum of loyal and general tourists exceeds the capacity, it is necessary to specify how many tourists of each type will be accepted. Here, we simply assume that they are randomly accepted in proportion to their numbers among the total number of tourists until all the accommodation facilities are filled. Let $b$ be the economic benefit per realized tourist.

$$
\text { If } C \leq x+y \text {, }
$$

The number of realized loyal tourists is $C x /(x+y)$.

The number of realized general tourists is $C y /(x+y)$. (7b)

Economic benefit is $b C$.

$$
\text { If } C \geq x+y,
$$

The number of realized loyal tourists is $x$.

The number of realized general tourists is $y$.

Economic benefit is $b(x+y)$.

Let $\phi$ be the net benefit to the local community after costs are taken into account. The expected net benefit is $\phi=b$. $\min [C, x+y]-k_{c} C-k_{q} Q$.

The arithmetic average of $\phi$ is

$E[\phi]=b\left[(1-p) \cdot \min \left[C, x+N_{h}\right]+p \cdot \min \left[C, x+N_{l}\right]\right]-k_{c} C-k_{q} Q$.

In Appendix 1, we show that the optimal solution can be obtained by considering only the following case:

Inequality $W(C, Q)+N_{l} \leq C \leq W(C, Q)+N_{h}$ holds, and

$E[\phi]=b\left[(1-p) C+p \cdot\left(W(C, Q)+N_{l}\right)\right]-k_{c} C-k_{q} Q$.

Our analysis of Eq. (9) is given in Appendix 1. The results are illustrated in Fig. 2, which are contour maps of function (9) for three different values. Horizontal and vertical axes are the investment in environmental quality $Q$ and accommodation capacity $C$, respectively, and the dashed lines are used to determine the optimal choices of $C$ and $Q$.

Figure 2a illustrates the case in which the maximum net benefit is achieved when $C=W(C, Q)+N_{h}$. Here, the policy is chosen by assuming that the maximum number of general tourists will come. We may call this the "optimistic policy." In contrast, if the maximum net benefit is achieved when $C=W(C$, $Q)+N_{l}$ (Fig. 2d), then, the policy is chosen by assuming the minimum number of general tourists. We may call this the "pessimistic policy." Figure $2 \mathrm{~b}$ and $\mathrm{c}$ illustrate cases with two local optima: one optimum is an optimistic policy and the other is a pessimistic policy. At the first local optimum, a large investment in accommodation capacity and a relatively low investment in quality improvement are made. At the second local optimum, a small investment in accommodation capacity but a high investment in quality improvement is made. The global optimum illustrated in Fig. $2 a$ and $b$ is an optimistic policy, whereas that illustrated in Fig. 2c and d is a pessimistic policy.

\section{Local optima lie on the boundaries of the region}

We found that local optima always lie on the boundaries of the region between $C=W(C, Q)+N_{h}$ and $C=W(C, Q)+N_{l}$. We have never seen a case in which a local optimum exists within the region surrounded by $C=W(C, Q)+N_{h}$ and $C=W(C$, $Q)+N_{l}$. This condition can be proved mathematically as follows. If there were an internal optimum, then, the second derivative of $E[\phi]$ would form a Hessian matrix:

$$
\left[\begin{array}{cc}
b p \frac{\partial^{2} W}{\partial C^{2}} & b p \frac{\partial^{2} W}{\partial C \partial Q} \\
b p \frac{\partial^{2} W}{\partial C \partial Q} & b p \frac{\partial^{2} W}{\partial Q^{2}}
\end{array}\right]
$$

which should have two negative eigenvalues. However, from Eq. (2), $\frac{\partial^{2} \mathrm{~W}}{\partial \mathrm{C}^{2}}>0$ and $\frac{\partial^{2} \mathrm{~W}}{\partial \mathrm{Q}^{2}}<0$ hold, which implies that the Hessian matrix would have one positive and one negative eigenvalue. Therefore, an internal point cannot be a local optimum of $E[\phi]$.

The optimal solution often involves some investment in environmental quality improvement i.e., $Q>0$ holds at the local optimum of the expected profitability $E[\phi]$. With positive $k_{Q}$, very large $N_{h}$, large $m$, and very small $w_{0}$, very little investment in environmental quality $(Q \approx 0)$ should be made (Fig. 3e). When $Q=0$, the net benefit increases with $C$ and is optimal only at $C=W(C, Q)+N_{h}$.

Thus, we can conclude that the local optimum must be either the optimistic or the pessimistic policy.

\section{Parameter dependence}

According to the mathematical analysis presented in the "Local optima lie on the boundaries of the region" section, the global optimum lies either on $C=W(C, Q)+N_{h}$ (optimistic policy) or on $C=W(C, Q)+N_{l}$ (pessimistic policy). According to the numerical simulation results, we classified the four possible cases as follows:

(Case 1) There exists a local optimum that is an optimistic policy, and it is also the global optimum. No local optimum that is a pessimistic policy exists (see Fig. 2a).

(Case 2) There exist two local optima: one is an optimistic policy, and the other is a pessimistic policy. The net benefit of the local optimum that is the optimistic policy is higher; therefore, it is the global optimum (see Fig. 2b). 
a

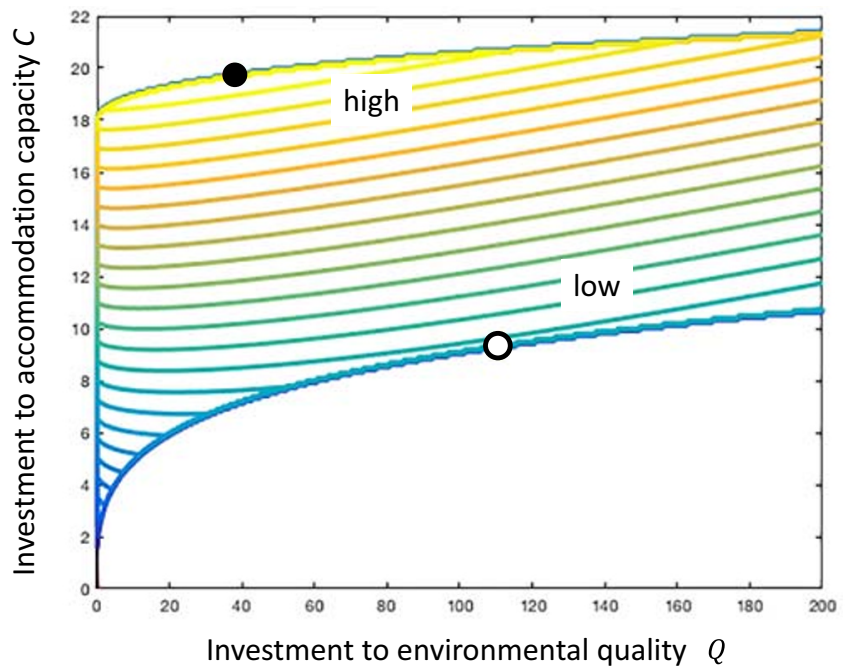

b

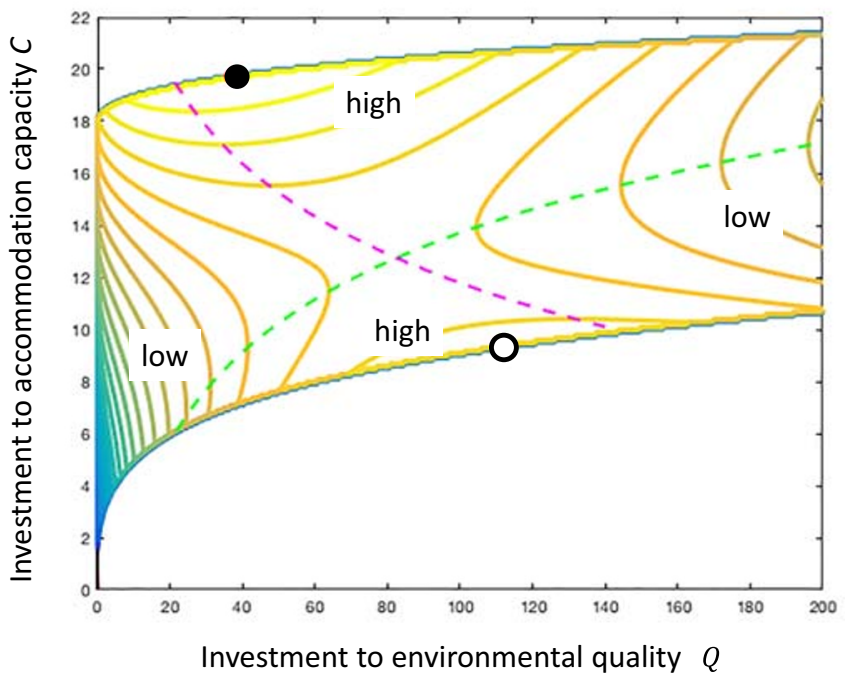

C

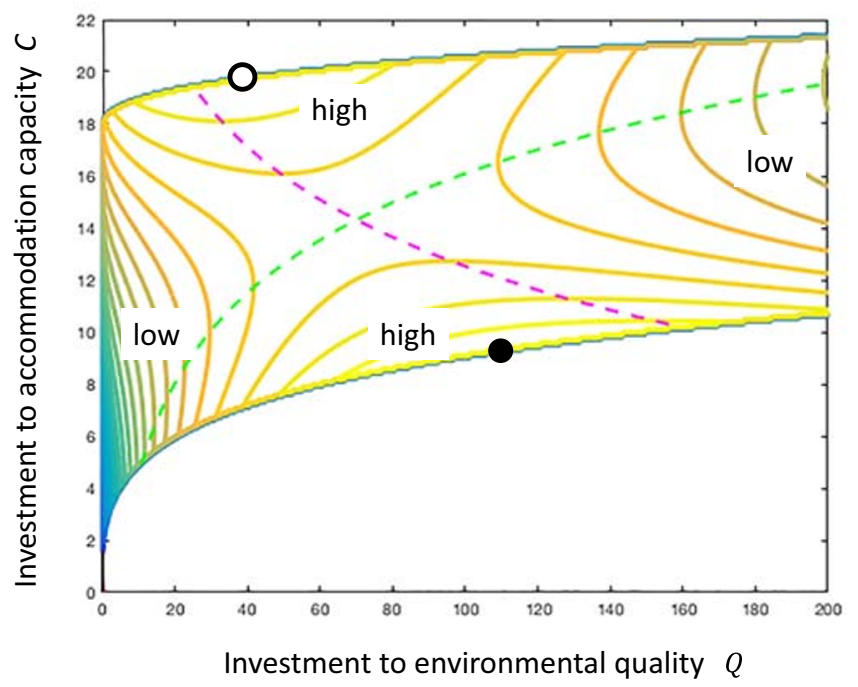

d

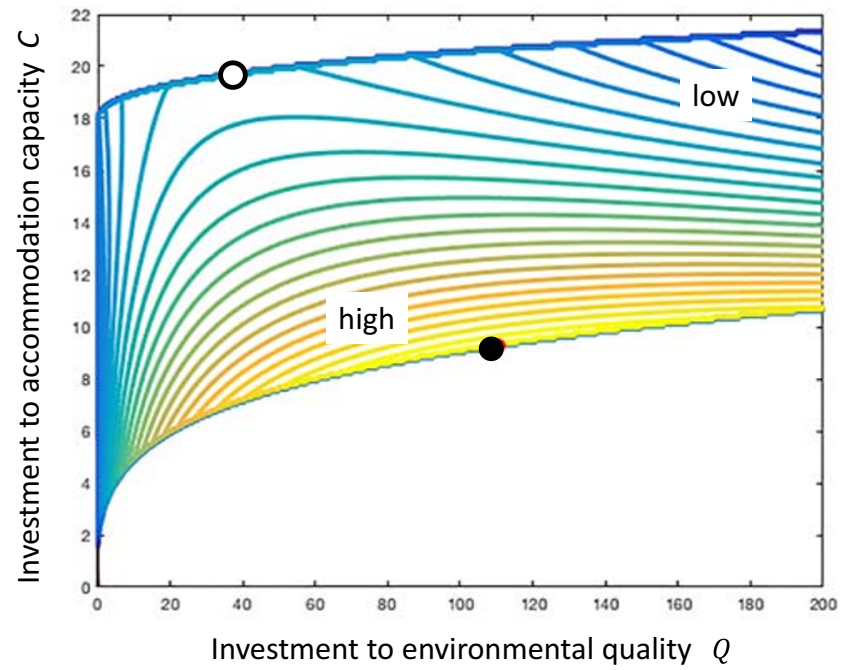

e

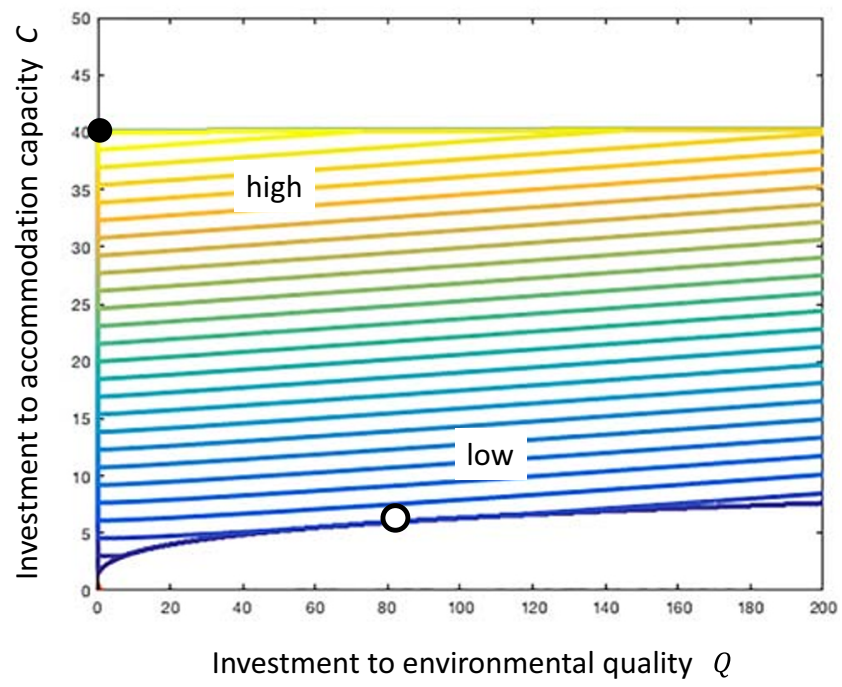


Fig. 2 The optimal choices of $C$ and $Q$. Thin curves are $C=W(C, Q)+$ $N_{h}$ and $C=W(C, Q)+N_{l}$. Thick curves are the expected benefit contours. a Case 1 . The global optimum (filled circle) lies on the boundary with a large number of general tourists defined by $C=W(C, Q)+N_{h}$, which is an optimistic strategy. There is no local optimum along curve $C=W(C, Q)+$ $N_{l}$. b Case 2. The global optimum (filled circle) is an optimistic strategy, but there is a local optimum (open circle) along the curve with a low number of general tourists defined by $C=W(C, Q)+N_{l}$. Green broken curve and red broken curves are for Eqs. (A.2a) and (A.2b), respectively. See Appendix 1 for their explanation. c Case 3. The global optimum is a pessimistic strategy, but there is a local optimum (open circle) that is an optimistic policy. d Case 4. The global optimum (closed circle) is a pessimistic strategy, and there is no local optimum that is an optimistic policy. e The global optimum is achieved when $Q=0$, and this point is on curve $C=W(C, Q)+N_{h}$ with a high number of general tourists. Therefore, this is an example of case 1. Other parameters are $p=0.1$ in (a), $p=0.65$ in (b), $p=0.7$ in (c), $p=0.9$ in (d), and $p=0.1, N_{h}=40$, and $w_{0}=1$ in (e). In addition, $N_{h}=18, N_{l}=1, w_{0}=2, m=0.1, b=5$, and $k_{Q}=k_{C}=0.1$

(Case 3) As in case 2, there exist two local optima, and one is an optimistic policy and the other is a pessimistic policy. However, the local optimum that is the pessimistic policy achieves a higher net benefit and is therefore the global optimum (see Fig. 2c).

(Case 4) There exists a local optimum that is a pessimistic policy, and it is also the global optimum. No local optimum that is an optimistic policy exists (see Fig. 2d).

We can distinguish these cases numerically. We first search for the optimum along $C=W(C, Q)+N_{h}$, and then determine whether it is a local optimum. We also search for the optimum along $C=W(C, Q)+N_{l}$, and determine if it is a local optimum. If both are local optima, then we compare the net benefit between them and identify the one with the higher net benefit as the global optimum. In Fig. $2 \mathrm{a}-\mathrm{d}$, the filled circles are global optima, and the open circles are optima along the curve but not the global optimum.

We examined the parameter dependence of the system by noting first whether the global optimum was an optimistic policy (cases 1 and 2) or a pessimistic policy (cases 3 and 4). We then examined combinations of parameters by choosing parameter values randomly from a uniform distribution of each parameter.

Figure $3 b-f$ show, for the four cases, the number of parameter combinations in which the global optimum is an optimistic policy (cases 1 and 2), and the number of combinations in which the global optimum is a pessimistic policy (cases 3 and 4) when one parameter ( $p, w_{0}, m, N_{h}$, or $N_{l}$, indicated on the horizontal axis) is fixed. The global optimum is more likely to be an optimistic policy as $p$ increases (Fig. 3b). Therefore, a higher probability of a general tourist collapse $p$ discourages optimistic policies (i.e., encourages pessimistic policies). Similarly, with higher $w_{0}$, lower $m$, and lower $N_{h}$ (Fig. 3c-e, respectively), the global optimum is more likely to be an optimistic policy. The value of $N_{l}$, however, has little effect on outcome (Fig. 3f).

The distribution of the optimal $(Q, C)$ that achieves the largest expected benefit is shown in Fig. $3 \mathrm{~g}$. Red circles indicate parameter combinations for which the global optimum is an optimistic policy (cases 1 and 2), whereas green circles indicate results for which the global optimum is a pessimistic policy (cases 3 and 4).

When $Q=0$, all global optima are optimistic (i.e., $C=$ $W(C, 0)+N_{h}$ holds), which is consistent with the conclusion in the last section.

The expected number of general tourists is the maximum number $N_{h}$ multiplied by the probability of its realization $1-p$. If $N_{h}$ becomes very large and $1-p$ becomes very small while their product is held constant, then, the optimistic policy becomes more risky. In the current model, relying on a very small probability of a huge success would not be adopted as an optimal policy. We compared the ratio of the optimal accommodation capacity with the maximum number of general tourists, $C^{*} / N_{h}$, against $N_{h}$ (Fig. 4 ). As $N_{h}$ becomes very large and $C^{*}$ also becomes large, the contribution from loyal tourists $W(C, Q)$ becomes negligible. Then, the optimistic policy is $C^{*} / N_{h}$ close to 1 , and the pessimistic policy is $C^{*} / N_{h}$ close to $N_{l} / N_{h}$, which must be smaller than 1 . The optimal policy is an optimistic policy when $N_{h} \leq 80$, but a pessimistic policy when $N_{h} \geq 90$. As $N_{h}$ becomes larger, $1-p$ becomes small, and another constraint is given by $b(1-p)>k_{Q}$, because large terms of Eq. (9) become $b(1-p) C-k_{Q} C$. Therefore, $1-p$ must be larger than approximately $k_{Q} / b$.

\section{Zoning}

Tourism site management may introduce site zoning. In our model, the satisfaction level of loyal tourists $W(C, Q)$ decreases as the number of hotels and other accommodations $C$ increases, but it is necessary for there to be enough accommodations to accept many loyal tourists. A more efficient use of the land is to split the site into two zones. Zone 1 has many hotels, restaurants, and other tourist facilities, whereas zone 2 has a minimal number of facilities, but efforts are made to increase the satisfaction level $W\left(C_{2}, Q_{2}\right)$ by enhancing environmental quality.

We formalize the model by defining zones 1 and 2 with capacities $C_{1}$ and $C_{2}$. The two kinds of tourists are local tourists $x$ and general tourists $y$. Assuming that tourists are assigned to either zone 1 or zone 2 randomly, we have

[1] If $x+y \geq C_{1}+C_{2}$, then 

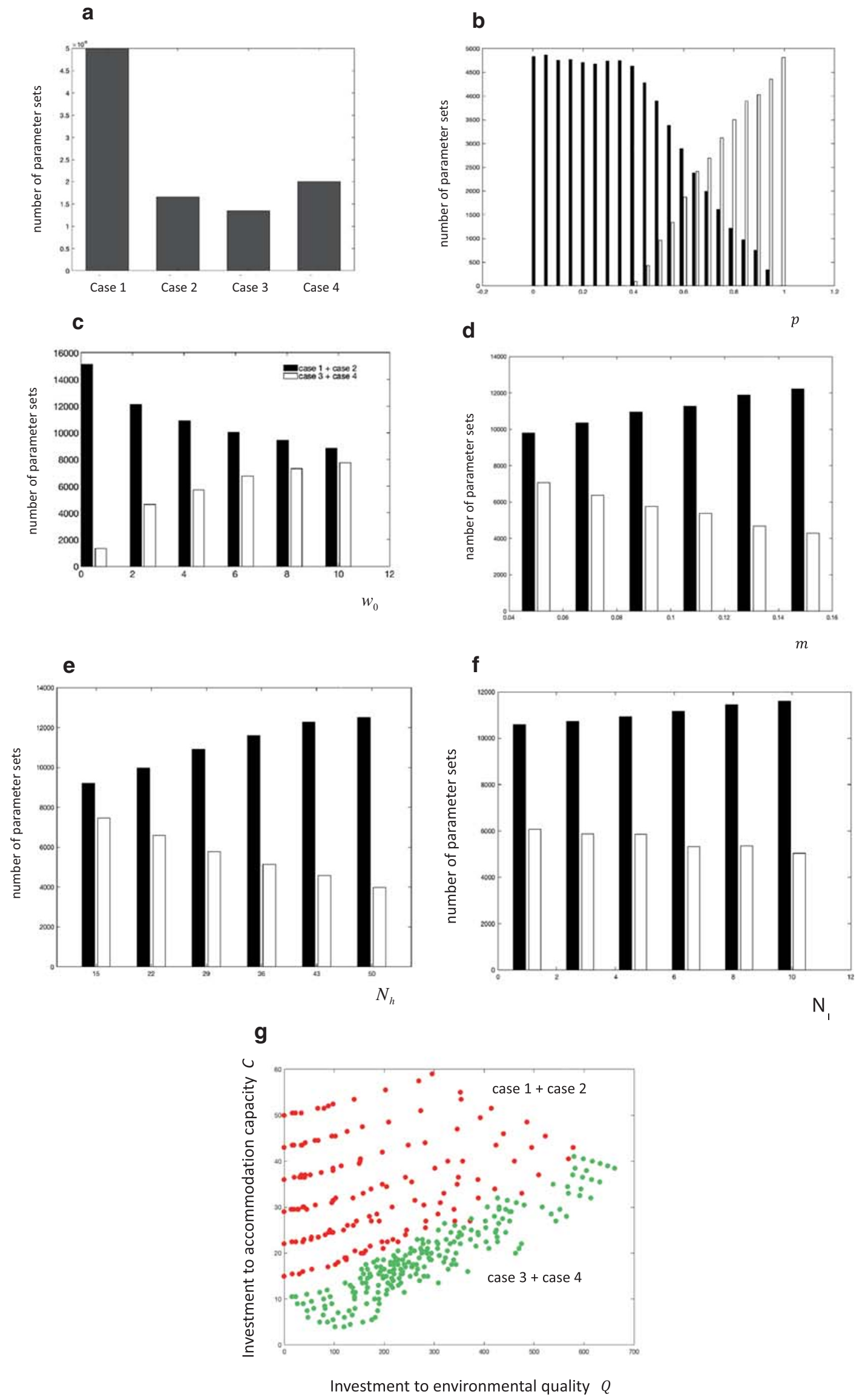
Fig. 3 Parameter dependence of the fraction of two cases when the global optimum is the optimistic policy, and the fraction of the other two cases when the global optimum is pessimistic. Parameters were chosen randomly and independently from a uniform distribution. The total number of parameter combinations was $10^{5}$. a Number of parameter sets among the four cases (see text for explanation of the four cases). $\mathbf{b}$, $\mathbf{c}, \mathbf{d}, \mathbf{e}$, and $\mathbf{f}$ The number of parameter sets when the global optimal is optimistic (cases 1 and 2 combined; filled bars), and the number of parameter sets when the global optimal is pessimistic (cases 3 and 4; open bars) when $p, w_{0}, m, N_{h}$, and $N_{l}$, respectively, are fixed. The fraction in cases 1 and 2 (an optimistic policy is optimal) increases with $m$ and $N_{h}$, but decreases with $w_{0}$ and $p$, where it is mostly independent of $N_{l}$. g Distribution of the optimal choices of $(Q, C) . C^{*}$ and $Q^{*}$ are plotted (red circles are for cases 1 and 2 and green circles for cases 3 and 4 ). The range of values of the other parameters is $w_{0}$ between 0.5 and $10 ; m$ between 0.05 and $0.15 ; p$ between 0.01 and $0.99 ; N_{h}$ between 15 and 50; and $N_{l}$ between 1 and 10. In addition, $k_{C}=0.1, k_{Q}=0.1$, and $b=10$

$$
\begin{aligned}
& R_{x 1}=C_{1} \frac{x}{x+y}, R_{x 2}=C_{2} \frac{x}{x+y}, R_{x}=\left(C_{1}+C_{2}\right) \frac{x}{x+y}, \\
& R_{y 1}=C_{1} \frac{y}{x+y}, R_{y 2}=C_{2} \frac{y}{x+y}, R_{y}=\left(C_{1}+C_{2}\right) \frac{y}{x+y} .
\end{aligned}
$$

[2] If $x+y \leq C_{1}+C_{2}$, then

$$
\begin{aligned}
& R_{x 1}=\frac{C_{1}}{C_{1}+C_{2}} x, R_{x 2}=\frac{C_{2}}{C_{1}+C_{2}} x, R_{x}=x, \\
& R_{y 1}=\frac{C_{1}}{C_{1}+C_{2}} y, R_{y 2}=\frac{C_{2}}{C_{1}+C_{2}} y, R_{y}=y .
\end{aligned}
$$

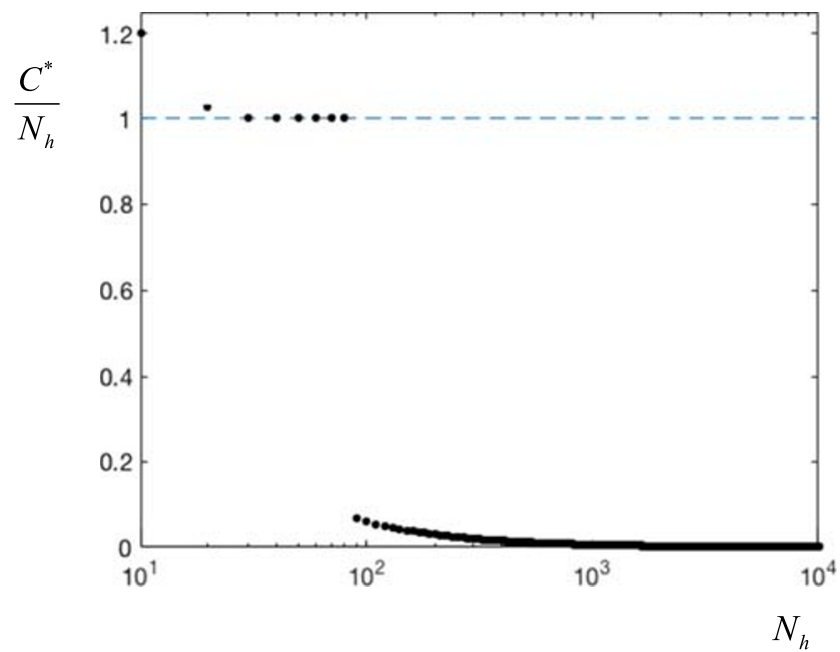

Fig. 4 The optimal policy choice. The vertical axis shows the ratio of the optimal accommodation capacity $C^{*}$ to the maximum number of general tourists $N_{h}$. The horizontal axis shows $N_{h}$ on a logarithmic scale. The expected number of general tourists $N_{h}(1-p)$ is fixed at 5. As $N_{h}$ becomes greater, the probability that the expected number will be realized becomes smaller, and the situation becomes more risky. When $N_{h} \leq 80$, the global optimum is the optimistic policy but when $N_{h} \geq 90$, the global optimum is the pessimistic policy. The area in which both solutions are local optima is also indicated. Other parameters are $N_{l}=1, w_{0}=1$, $m=0.1, b=10$, and $k_{Q}=k_{C}=0.1$
Situations [1] and [2] correspond to the case in which the realized number of tourists is limited by the accommodation capacity and by the number of potential tourists, respectively.

The potential number of loyal tourists changes over time, following the logistic equation $x^{\text {next }}=x+\delta x\{\overline{\bar{W}}-x\}$, where $\delta$ is turnover rate and $\overline{\bar{W}}=W\left(C_{1}, Q_{1}: C_{2}, Q_{2}\right)$ is the mean satisfaction level of loyal tourists. $\overline{\bar{W}}$ is the arithmetic average of the satisfaction of loyal tourists who recently visited two zones. The final equilibrium value is $x=\overline{\bar{W}}(C, Q)$.

If the quality difference between the two zones is large (e.g., $W\left(C_{2}, Q_{2}\right)$ is much larger than $W\left(C_{1}, Q_{1}\right)$ ), all of the visitors staying in both zones spend their time in the highquality zone and have satisfaction level $W\left(C_{2}, Q_{2}\right)$. However, those staying in the lower quality zone bear the additional cost of commuting between the two zones. Let $D_{F}$ be a factor less than 1 , which we call the "distance factor." Then,

$\overline{\bar{W}}(C, Q)=\frac{C_{1} D_{F}+C_{2}}{C_{1}+C_{2}} W\left(C_{2}, Q_{2}\right)$.

If the distance between the two zones is large, the distance factor becomes much smaller than 1 and tourists staying in the two zones stay in their respective zones. The mean level of satisfaction is:

$\overline{\bar{W}}(C, Q)=\frac{C_{1}}{C_{1}+C_{2}} W\left(C_{1}, Q_{1}\right)+\frac{C_{2}}{C_{1}+C_{2}} W\left(C_{2}, Q_{2}\right)$.

We assume that the tourists who stay in accommodations in area 1 (with the lower quality environment) will choose zone to spend their time by comparing satisfaction levels between the two zones. Hence, the mean satisfaction level for all loyal tourists is Eq. (12a) if $D_{F} W\left(C_{2}, Q_{2}\right) \geq W\left(C_{1}, Q_{1}\right)$, and is Eq. (12b) if $D_{F} W\left(C_{2}, Q_{2}\right) \leq W\left(C_{1}, Q_{1}\right)$. If the distance between the two zones is small compared with the quality difference between them, then, Eq. (12a) should hold, because all loyal visitors staying in both zones spend their time in zone 2. However, the mean satisfaction level is lower than $W\left(C_{2}, Q_{2}\right)$ by the mean distance factor $\left\langle D_{F}\right\rangle=\frac{C_{1} D_{F}+C_{2}}{C_{1}+C_{2}}$, which is less than 1 .

The number of general tourists is a stochastic variable, as shown in Eqs. (6a) and (7b).

In Appendix 2, we analyze the situation described by Eq. (12a) (all tourists staying in zone 1 go spend their time in zone 2). This situation is plausible when the distance factor is not strong $\left(D_{F}\right.$ is not very much smaller than 1$)$. Then, the optimal choice is $Q_{1}=0$, and $C_{2}=0$ i.e., no accommodations should be built in zone 2 and no investment in environmental quality should be made in zone 1. In this situation, zone 1, with high accommodation pressure, is clearly separated from zone 2, which has enhanced environmental quality. 


\section{Discussion}

Since ecotourism is a potentially very useful method of maintaining biodiversity conservation and ecosystem management, understanding basic mechanisms realizing successful management is an important problem in conservation ecology. In this paper, we developed a simple model to know the optimal policy choice for development of an ecotourism site with respect to investments in accommodation capacity (including hotels, restaurants, and transportation facilities) and enhancing the quality of the natural and cultural attractions of the site (including biodiversity, landscape, and cultural activities).

As shown in Jeju Island, massive numbers of tourists visit in groups and bring large economic benefit, but they may harm the sustainability of the natural environment (Kang et al. 2013, 2018; Kim and Jin 2018). Moreover, the number of tourists may fluctuate unpredictably (An et al. 2017; Kim and Kim 2017; Park et al. 2019), for example, if changing popular trends cause tourists to be attracted to a competing tourism site.

Ecotourism is an alternative type of tourism. It focuses on loyal tourists, who become fond of the site and are likely to come back many times in the future. In-depth interviews with DIY travelers show that increases in the accommodation capacity discourage loyal tourists. Due to the island's loss of authenticity in recent years, they are reluctant to confirm any intention to return, even though they are fond of the island and its attractions (Jeju Special Self-Governing Province 2017).

In this paper, we modeled this situation by noting that tourists are heterogeneous: "general tourists" are abundant but they will not come back to the focal site and their future numbers are highly unpredictable; in contrast, "loyal tourists" are repeaters, who tend to return to the site. Loyal tourists are attracted by features such as the endemic species, natural history, landscape, authentic culture, and history of a site. We then searched for the optimal policy with respect to investments in expanding the accommodation capacity and improvements in the natural and cultural quality of the site that would maximize the average future net benefit realized by the whole community.

\section{Optimistic policy and pessimistic policy}

One conclusion of our model is that the optimal combination of investments in accommodation capacity $C$ and environmental quality $Q$ always lies either on the curve that assumes the maximum number of tourists comes, or on the one that assumes a minimum number of tourists. We may call combinations that lie on the former curve optimistic policies. They target the general tourists, and they tend to have a large investment in accommodation capacity but a relatively low investment in environmental quality improvement. In contrast, we may call combinations of investment that lie in the latter curve pessimistic policies. These target the loyal tourists, and they tend to have a small investment in the accommodation capacity but a relatively low investment in environmental quality improvement. We provided the mathematical proof that the optimal will never lie in the interior region between these two curves for any function $W(C, Q)$ that satisfies Eq. (2) by examining the eigenvalues of the Hessian matrix.

When $m$ and $N_{h}$ are large and $w_{0}$ and $N_{l}$ are small, the optimal policy is likely to be an optimistic policy, whereas when $m$ and $N_{h}$ are small and $w_{0}$ and $N_{l}$ are large, the optimal policy is likely to be a pessimistic policy. This result is plausible because a pessimistic policy should be implemented if loyal tourists are more important.

\section{Zoning}

We also examined the situation with site zoning. If the site can be separated into two nearby zones, then, all accommodations should be concentrated in one zone, and investment in environmental improvements in the second zone should be high. If the zones are too far apart, however, zoning will not work, because tourists may not be willing to stay in the first zone and make daily visits to the second zone. In that situation, sufficient accommodations must be provided at the site without much reducing its attractiveness to loyal tourists. Clearly, whether zoning is feasible depends on the available transportation, among many other factors.

Zoning on Jeju Island has been discussed as a way to implement UNESCO Biosphere Reserve guidelines and achieve sustainable development management. These guidelines suggest three zones, each with a different strategy: a core zone where the focus is on conservation, a buffer zone where sound ecological practices are emphasized, and a transition zone where economic and human development activities take place (Ishwaran et al. 2008; UNESCO 2008). These guidelines are not legally binding, so development plans can be implemented even in the buffer zone or in an area close to the core zone. For example, over 69 forest bird species reside on oreums on Jeju Island, including nine nationally protected species, yet half of the oreums are being developed for grazing, plantations, and recreational areas such as resorts and golf courses, or are at severe risk for such development (Nam et al. 2019).

\section{Future study}

When the high number of general tourists $N_{h}$ is large and the probability of its collapse $p$ is high (i.e., close to 1), then, we must choose either the optimistic policy that assumes a large number of general tourists or the pessimistic policy that assumes a minimal number of general tourists - nothing in between would be optimal. 
As the maximum number of tourists $N_{h}$ to the site becomes larger, the optimal policy that maximizes the expected average net benefit might be the optimistic policy, even if the probability $1-p$ that the assumption of a large number of future tourist will be realized is small. However, small $1-p$ implies that in most years, the outcome will be a disaster with many empty hotels and high unemployment, although there is a small probability of a very great success. In our model, for an optimistic policy is to be the optimal policy, there exists a lower limit of $1-p$, which is approximately $k_{Q} / b$ (see Fig. 4). However, at this lower limit, the probability is still too small for this optimistic policy to be a realistic choice as the tourism development policy of the whole community. Thus, we probably need to incorporate a "risk avoidance" parameter into the criteria used to choose the policy. Instead of maximizing the arithmetic average of the net benefit, we should maximize the geometric average of the future net benefit. In such a model, a policy with a large variance in economic benefit would jeopardize the future net benefit.

We have been analyzing the case in which the number of general tourists is two levels $\left(N_{h}\right.$ or $\left.N_{l}\right)$. This is the assumption to simplify the model. However, in the future extension examining the case in which the level of general tourists has a more general probability distribution.

In this paper, we consider only the economic benefit of the local communities as the criteria for the desirability of different policies. However, we may be able to incorporate the viewpoint of other aspects, such as biodiversity conservation or SDGs etc. in designing the evaluation of policies.

Finally, in this paper, we assumed that general tourists and loyal tourists are different people. However, we may be able to make transition from general to loyal tourists by environmental education enhancing public understanding of the importance of biodiversity conservation. This is an important theme for future theoretical studies.

Acknowledgements We thank the D. Ikeda and Y-K. Jeong for the useful comments.

Funding information This work was supported by a Grant-in-Aid for Scientific Research (no. 19K16231) from the Japan Society for the Promotion of Science to JHL.

\section{Appendix 1}

We consider the optimization of Eq. (9). We have three cases:

Case 1: Inequality $W(C, Q)+N_{l} \leq C \leq W(C, Q)+N_{h}$ holds, and

$$
E[\phi]=b\left[(1-p) C+p \cdot\left(W(C, Q)+N_{l}\right)\right]-k_{c} C-k_{q} Q .
$$

Case 2: Inequality $C<W(C, Q)+N_{l}$ holds, and

$E[\phi]=b C-k_{c} C-k_{q} Q$.

Assuming $b>k_{c}$, then $E[\phi]$ is an increasing function of $C$; hence, the optimal choice is within case 1 .

Case 3: If $C>W(C, Q)+N_{h}$

$E[\phi]=b\left[W(C, Q)+(1-p) N_{h}+p N_{l}\right]-k_{c} C-k_{q} Q$.

If Eq. (A.1c) is also a decreasing function of $C$, then, the optimal choice is within case 1 . Hence, we can conclude that the optimal choice must be within case 1 .

As discussed in the main text, the Hessian matrix of $E[\phi]$ as a function of $C$ and $Q$ has one positive and one negative eigenvalues (see Eq. (10)). This implies that no inner point of the area surrounded by the two curves can be a local optimum. Hence, we can search for the local and/or global optima along the boundary of the area, which is given by two curves: $C=W(C, Q)+N_{h}$ and $C=W(C, Q)+N_{l}$. To obtain the local optimum mathematically, we may use Lagrange's multiplier method: we search for the maximum of $E[\phi]$ under the constraint given by the boundaries. Alternatively, we can simply search for the local maximum along the boundary numerically, by examining the value of $E[\phi]$ along the boundaries. There must be one for each of the two curves. However, these may or may not be local maxima. To confirm them as a local maximum, we need to calculate $E[\phi]$ at the candidate point and at an inner point close to it. If the focal point has $E[\phi]$ larger than the nearby inner point, it is a local optimum. Otherwise, the candidate point is not a local optimum (although it is the local maximum along the boundary). If we obtained multiple local optima, we then identify the global optimum by comparing $E[\phi]$ between them.

In each parts of Fig. 2, the global optimum is indicated by a solid circle. We can confirm this numerically. Local optima are indicated by open circles in Fig. 2. In Fig. 2b and c, green and red broken curves are calculated from $\partial E[\phi] / \partial C=0$ and $\partial E[\phi] / \partial Q=0$, which are given by

$b(1-p)+b p \frac{\partial W}{\partial C}-k_{c}=0$,

$b p \frac{\partial W}{\partial Q}-k_{Q}=0$,

respectively. Green broken curves indicate the location of "valley."

\section{Appendix 2}

The problem is formalized in a very similar manner to the model analyzed in Appendix 1. We here focus on the situation in which the two zones are not very far apart (distance factor 
$D_{f}$ is smaller than 1 but not very small). Tourists staying in one zone can enjoy the landscape and natural attractions in the other zone. Specifically, we assume Eq. (12a). The average net benefit is

$$
\begin{aligned}
E[\phi]= & (1-p) b \cdot \min \left[\overline{\bar{W}}\left(C_{2}, Q_{2}\right)+N_{h}, C_{1}+C_{2}\right] \\
& +p b \cdot \min \left[\overline{\bar{W}}, C_{1}+C_{2}\right]-k_{c}\left(C_{1}+C_{2}\right)-k_{Q}\left(Q_{1}+Q_{2}\right) .
\end{aligned}
$$

Again, we identify three cases as in Appendix 1.

Case 1: $\overline{\bar{W}}\left(C_{2}, Q_{2}\right)+N_{l} \leq C_{1}+C_{2} \leq \overline{\bar{W}}\left(C_{2}, Q_{2}\right)+N_{h}$ holds and we have

$$
\begin{aligned}
E[\phi]= & (1-p) b \cdot\left(C_{1}+C_{2}\right)+p b \cdot\left(\overline{\bar{W}}\left(C_{2}, Q_{2}\right)+N_{l}\right) \\
& -k_{c}\left(C_{1}+C_{2}\right)-k_{Q}\left(Q_{1}+Q_{2}\right) .
\end{aligned}
$$

Eq. (B.2) decreases with $Q_{1}$, and $Q_{1}=0$ is the optimal choice. Because it increases with $C_{1}, C_{1}+C_{2}=\overline{\bar{W}}\left(C_{2}, Q_{2}\right)+N_{h}$ must hold at the optimum point. Then, by replacing this, Eq. (B.2) becomes.

$E[\phi]=\left((1-p) b+p b-k_{c}\right) \cdot \overline{\bar{W}}\left(C_{2}, Q_{2}\right)-k_{Q} Q_{2}+[\ldots]$

This equation decreases with $C_{2}$; hence $C_{2}=0$ is the optimum. Thus, $Q_{2}$ is determined by

$\left(b-k_{c}\right) \cdot \frac{\partial}{\partial Q_{2}} \overline{\bar{W}}\left(0, Q_{2}\right)=k_{Q}$.

Case 2: $C_{1}+C_{2} \leq \overline{\bar{W}}\left(C_{2}, Q_{2}\right)+N_{l}$ holds and we have

$E[\phi]=\left(b-k_{c}\right) \cdot\left(C_{1}+C_{2}\right)-k_{Q}\left(Q_{1}+Q_{2}\right)$.

Since $b>k_{c}, C_{1}+C_{2}=\overline{\bar{W}}\left(C_{2}, Q_{2}\right)+N_{l}$ holds at the optimum. Hence, the search for the optimum is the same as in Case 1.

Case 3: $C_{1}+C_{2} \geq \overline{\bar{W}}\left(Q_{2}, C_{2}\right)+N_{h}$ holds and we have

$$
\begin{aligned}
E[\phi]= & b \cdot\left(\overline{\bar{W}}\left(C_{2}, Q_{2}\right)+(1-p) N_{h}+p N_{l}\right)-k_{c}\left(C_{1}+C_{2}\right) \\
& -k_{Q}\left(Q_{1}+Q_{2}\right) .
\end{aligned}
$$

$E[\phi]$ is a decreasing function of both $C_{1}$ and $C_{2}$; hence, the optimum in this case is $C_{1}+C_{2}=\overline{\bar{W}}\left(C_{2}, Q_{2}\right)+N_{h}$, and the search for the optimal choice is the same as in Case 1.

In summary, the optimal choice should be achieved when $Q_{1}=0$ (no environmental improvement in zone 1) and $C_{2}=0$ (no accommodation in zone 2). This result implies two clearly separated zones. The other two variables, $Q_{2}$ and $C_{1}$, are determined by using Eq. (B.3) and $C_{1}=\overline{\bar{W}}\left(0, Q_{2}\right)+N_{h}$, respectively. For the example of $\overline{\bar{W}}(C, Q)=\left\langle D_{F}\right\rangle w_{o} e^{-m C} \sqrt{Q}$, we have

$Q_{2}=\left(\frac{\left(b-k_{C}\right) w_{0}}{2 k_{Q}} D_{F}\right)^{2}$,

$C_{1}=N_{h}+\frac{b-k_{C}}{2 k_{Q}}\left(w_{0} D_{F}\right)^{2}$,

which can be large if transportation between the two zones is not costly (i.e., $\left\langle D_{F}\right\rangle$ is not small), and if the cost of the environmental improvement $k_{Q}$ is small.

Open Access This article is licensed under a Creative Commons Attribution 4.0 International License, which permits use, sharing, adaptation, distribution and reproduction in any medium or format, as long as you give appropriate credit to the original author(s) and the source, provide a link to the Creative Commons licence, and indicate if changes were made. The images or other third party material in this article are included in the article's Creative Commons licence, unless indicated otherwise in a credit line to the material. If material is not included in the article's Creative Commons licence and your intended use is not permitted by statutory regulation or exceeds the permitted use, you will need to obtain permission directly from the copyright holder. To view a copy of this licence, visit http://creativecommons.org/licenses/by/4.0/.

\section{References}

An S-H, Juan Y-X, Lee C-K (2017) A case study of the effect of political conflict between countries on the travel decision-making process: focusing on the THAAD political conflict between South Korea and China. Korean Cult Tour Instit 67:63-38

Ceballos-Lascuráin H (1996) Tourism, ecotourism and protected areas: the state of nature-based tourism around the world and guidelines for its development. IUCN publications, Cambridge 301

Choi K-W, Suh Y-K (2019) Measuring sustainable tourism - focused on Jeju Island. J Reg Stud 27(1):107-121

Chung JY, Whang T (2011) The impact of low cost carriers on Korean Island tourism. J Transp Geogr 19(6):1335-1340

Goodwin H (1996) In pursuit of ecotourism. Biodivers Conserv 5(3): 277-291

Ishwaran N, Persic A, Tri NH (2008) Concept and practice. The case of UNESCO biosphere reserves. Int J Environ Sustain Dev 7(2):118131

Jeju Branch of the Bank of Korea (2018) Analysis of the economic ripple effect of the tourists on the regional economy of Jeju province. Jeju Econ Brief:2018-2016

Jeju Special Self-Governing Province \& Jeju Tourism Organization (2017) Visitor survey. https://www.jeju.go.kr/group/part28/refer. $\mathrm{htm}$; jsessionid=NQcIXEps00KV51f2r2CioRUNUWpF NyzfSSAvxeB2Z7RIaUP1M24H11IB3h7Urvfq.was2_servlet_ engine7?act=view\&seq=1092389. Accessed 30 Oct 2019

Jeju Special Self-Governing Province \& Jeju Tourism Organization (2018) Report for tourism capacity management of Jeju island. https://www.ijto.or.kr/korean/Bd/view.php?btable=policy\&bno= 36\&pds_skin $=\& p=1 \& l$ cate $=$. Accessed 30 Oct 2019 
Kang EJ, Scott N, Lee TJ, Ballantyne R (2012) Benefits of visiting a 'dark tourism's site: the case of the Jeju April 3rd Peace Park, Korea. Tour Manag 33(2):257-265

Kang H-G, Kim C-S, Kim E-S (2013) Human influence, regeneration, and conservation of the Gotjawal forests in Jeju Island, Korea. J Marine Island Cult 2:85-92

Kang JY, Choi I-S, Jeon D-U (2018) Dynamic analysis of solid waste management systems in Jeju. Korean Syst Dyn Res 19(3):49-75

Ki JS, Jeon Y, Ryu CK (2016) A signification and meaning of Geomunoreum as a representative geosite of global Geoparks. J Geol Soc Korea 52:763-774 (in Korean)

Kim J-K (2018) An impact of THAAD controversy on Korean travelers: intention of revisiting China - emphasis on demographic characteristics and travel patterns. J Tour Leis Res 30(1):167-184

Kim H-S, Jin S-H (2018) An analysis of agenda setting and regulatory politics in the process of Jeju UNESCO world natural heritage. Korean Environ Policy 26(4):147-179

Kim D-H, Kim Y-K (2017) Importance performance analysis on selection attributes of travel destination by FIT Chinese travelers with Bona fide travel purpose to Korea. Int J Tour Manag Sci 34(3):219-236

Kim H, Xiang Z, Fesenmaier DR (2014) Use of the internet for trip planning: a general analysis. J Travel Tour Mark 32:276-289

Latzko DA (2004) Tourism and fluctuations in the Hawaiian economy. J Tour Stud 15(2):67-72

Lee J-H, Iwasa Y (2011) Tourists and traditional divers in a common fishing ground. Ecol Econ 70:2350-2360

Luthe T, Wyss R (2014) Assessing and planning resilience in tourism. Tour Manag 44:161-163

Nam H-Y, Kim E-M, Choi C-Y, Kang C-W (2019) Avifauna of Gungdae Oreume and its seasonal changes in the Jeju Eastern Oreum Group in Jeju Island. Korea J Asia-Pac Biodivers (in press). https://doi.org/ 10.1016/j.japb.2019.06.002

Park G-Y, Han H-L, Choi S-D (2019) A comparative study on the changes in perceptions of Korea tourism in Chinese tourists before and after the THAAD using big data: focused on semantic network analysis. J Tour Leis Res 31(2):25-43

Suh Y-K, Jo J-I (2015) Development of sustainable tourism indicators focused on comparison of island \& non-island areas. J Reg Stud 23(3):91-110

Torquebiau E, Taylor R (2009) Natural resource management by rural citizens in developing countries: innovations still required. Biodivers Conserv 18:2537-2550. https://doi.org/10.1007/s10531009-9706-3

UNESCO (2008) Madrid action plan for biosphere reserves (20082013). UNESCO, Paris. http://unesdoc.unesco.org/images/0016/ 001633/163301e.pdf. Accessed 1 Dec 2019

UNWTO (2011) Tourism towards 2030: global overview. World Tourism Organization (UNWTO), Madrid. Access December 1, 2019. https://www.globalwellnesssummit.com/wp-content/uploads/ Industry-Research/Global/2011_UNWTO_Tourism_Towards_ 2030.pdf. Accessed 29 Oct 2019

UNWTO (2018) UNWTO tourism highlights 2018 edition. Access December 1, 2019. Retrieved from https://www.e-unwto. org/doi/pdf/10.18111/9789284419876

World Bank (2016) World development indicators: electricity production, sources, and access. Retrieved from http://wdi.worldbank.org/table/ 3.7. Accessed 1 Dec 2019

World Travel and Tourism Council (WTTC) (2016) Global tourism data gateway. Retrieved from http://www.wttc.org/datagateway . Accessed 1 Dec 2019

Xiang Z, Wang D, O'Leary J, Fesenmaier DR (2014) Adapting to the internet: trends in travelers' use of the web for trip planning. J Travel 54. https://doi.org/10.1177/0047287514522883

Xiang Z, Magnini VP, Fesenmaier DP (2015) Information technology and consumer behavior in travel and tourism: insights from travel planning using the internet. J Retail Consum Serv 22:244-249 